\title{
Evaluation of bioherbicidal potential of Carica papaya leaves
}

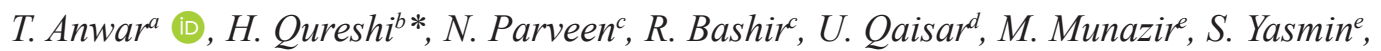 \\ Z. Basit, R. T. Mahmood, B. G. Nayyar ${ }^{h}$, S. Khan ${ }^{i}$, S. A. Khan', M. M. Qureshi ${ }^{k}$ and M. Wali ${ }^{i}$
}

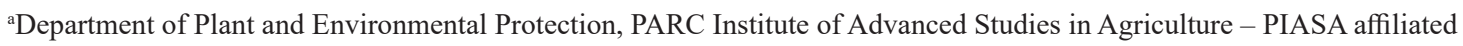
with The Agriculture University, Peshawar, National Agriculture Research Centre - NARC Campus, Park Road, Islamabad-44000, Pakistan

${ }^{b}$ Department of Botany, Rawalpindi Women University, Satellite Town, Rawalpindi-46300, Punjab, Pakistan

'Department of Botany, Government College University, Kotwali Road, Faisalabad-38000, Punjab, Pakistan

${ }^{\mathrm{d} S}$ School of Biological Sciences, University of The Punjab, Lahore-54000, Punjab, Pakistan

${ }^{e}$ Department of Botany, Government College for Women University, Kutchehry Road, Sialkot- 51310, Punjab, Pakistan

${ }_{\mathrm{f}}^{\mathrm{f}}$ Department of Mathematics and Statistics, Pir Mehr Ali Shah Arid Agriculture University, Rawalpindi-46300, Punjab, Pakistan

'Department of Biotechnology, Mirpur University of Science and Technology, Mirpur-10250, Azad Jammu \& Kashmir, Pakistan

hepartment of Botany, University of Sialkot, Sialkot-51310, Punjab, Pakistan

'Department of Environmental Science, Gomal University, Dera Ismail Khan-29050, Khyber Pakhtunkhwa, Pakistan

jDepartment of Earth and Environmental Science, Bahria University, Sector E-8, Islamabad-44000, Pakistan

${ }^{k}$ Department of Plant Breeding and Genetics, Gomal University, Dera Ismail Khan-29050, Khyber Pakhtunkhwa, Pakistan

*e-mail: humaqureshi8@gmail.com

Received: November 10, 2018 - Accepted: April 29, 2019 - Distributed: August 31, 2020

\begin{abstract}
Due to increased number of herbicide resistant weeds, it is needed to explore the allelopathic potential of plants as an alternative. The research was conducted to investigate allelopathic effects of Carica papaya L. leaf powder and aqueous extract on seeds as well as pre-germinated seeds of Avena fatua L., Helianthus annuus L., Rumex dentatus L., Zea mays L. and Triticum aestivum L. on filter paper and soil in Weed Management Program Laboratory, Department of Plant and Environmental Protection at PARC Institute of Advanced Studies in Agriculture, National Agriculture Research Centre, Islamabad, Pakistan. Germination percentage (\%), radicle length $(\mathrm{cm})$ and plumule length $(\mathrm{cm})$ were parameters observed for 'Plant leaf powder bioassay' and 'Aqueous extract method'. Most significant growth inhibition was observed in $A$. fatua seedlings in filter paper method. A. fatua radicle length was reduced by $C$. papaya aqueous extract (80\%) and leaf powder (89\%) bioassays. Plumule length was reduced under the influence of aqueous extract (57-73\%) and powdered material (59-77\%). The inhibitory effects on other test species were in sequence of $H$. annuus followed by $Z$. mays and $R$. dentatus. The aqueous extract showed non-significant effect on wheat seed germination, radicle and plumule growth. It is suggested that $C$. papaya aqueous extract can be used as source of weed management in wheat crop.
\end{abstract}

Keywords: allelopathy, bioherbicide, Rumex dentatus, Avena fatua, weed suppression.

\section{Avaliação do potencial de bioherbicida dase folhas de Carica papaya}

\section{Resumo}

Devido ao aumento do número de ervas daninhas resistentes aos herbicidas, é necessário explorar o potencial alelopático das plantas como uma alternativa. A pesquisa foi conduzida com o objetivo de investigar os efeitos alelopáticos do pó foliar de Carica papaya e do extrato aquoso das sementes, bem como das sementes pré-germinadas de Avena fatua, Helianthus annuus, Rumex dentatus, Zea mays e Triticum aestivum em papel de filtro e solo no Laboratório do Programa de Manejo de Ervas Daninhas, Departamento de Plantas e Proteção Ambiental do Instituto PARC de Estudos Avançados em Agricultura, Centro Nacional de Pesquisa Agrícola, Islamabad, Paquistão. A porcentagem de germinação (\%), o comprimento radicular e o comprimento da plúmula $(\mathrm{cm})$ foram os parâmetros observados para o 'Bioensaio de Pó de Folha de Planta' e o 'Método de Extração Aquoso'. A maior inibição do crescimento foi observada em mudas de A. fatua no método de papel de filtro. O comprimento radicular de $A$. fatua foi reduzido com os extratos aquosos de C. papaya (80\%) e pó de folhas (89\%). O comprimento das plúmulas foi reduzido sob a influência do extrato aquoso 
(57-73\%) e material em pó (59-77\%). Os efeitos inibitórios em outras espécies-teste foram na sequência de H. annuus seguido por $Z$. mays e $R$. dentatus. $\mathrm{O}$ extrato aquoso apresentou efeito não significativo na germinação das sementes de trigo, nos crescimentos radiculares e das plúmulas. Sugere-se que o extrato aquoso de C. papaya pode ser utilizado como fonte de manejo de plantas daninhas na cultura do trigo.

Palavras-chave: alelopatia, bioherbicida, Rumex dentatus, Avena fatua, supressão de plantas daninhas.

\section{Introduction}

Weeds are non-economic plants that reduce crop yield (Mukhtar et al., 2012). Weeds along resource competition with crops exhibit allelopathy (Batish et al., 2007). Allelopathy is natural tool to increase crop yield by weed control reducing utilization of synthetic herbicides (Alagesaboopathi, 2010). Studies have suggested allelochemicals as alternatives to synthetic herbicides for weed control (Khanh et al., 2006; Jamil et al., 2009). In Pakistan, weeds are serious problem in crop production. Pesticides have been utilized for weed management but most of them are hazardous to the environment and human health. If weeds are properly controlled, grain production in Pakistan can be increased up to $37 \%$ (Khanh et al., 2008). Allopathy has been mentioned in many plants releasing chemicals in environment from aerial or underground parts. The allelochemicals released into environment act on neighboring weeds, plants, microbes both stimulatory and inhibitory. Allelopathic weeds have advantages from agronomic point of view and can be used in weed management practices (Anwar et al., 2016). Allelopathy is 'detrimental effect of one plant on another through release of chemicals'. The interaction is mediated by allelopathic compounds released from donor affecting acceptor organisms. Allelopathic interaction among individuals of same species is 'intraspecific toxicity' or 'auto-allelopathy'. Contrarily, term 'tele-toxicity' is used when suspect species is taxonomically different from donor species (Garima and Devi, 2017). Allelopathic compounds produced by higher plants are mostly secondary metabolites. Phenolics, quinones, cyanogenic glycosides, organic acids, lactones and terpenes belong to active compounds. Table 1 represents brief overview of allelochemicals isolated from different plants (Qureshi and Arshad, 2017). Phenolic acids and terpenoids are common types of allelochemicals (Shankar et al., 2009). Allelochemicals from donor plant disrupt physiological processes e.g., photosynthesis, respiration, water and hormonal balance of recipient plant by inhibition of enzyme activity (Soltys et al., 2013). Allelochemicals are useful for weed management in agriculture to reduce dependency on commercial herbicides. An allelopathic crop can potentially be used to control weeds through its allelochemicals. Scopolin and Scopoletin isolated from Celtis laevigata suppress Amaranthus palmeri. Annona squamosa, Coffea arabica, C. papaya, and Tamarindus indica inhibit germination of Amaranthus spinosus. Eucalytus citriodora showed allelopathic effect on the growth of Digitaria pertenuis, Bidens pilosa, Setaria geniculate, Eragrostis cilianensis. Similarly, Eucalyptus citriodora inhibited the germination of $P$. hysterophorus (Garima and Devi, 2017).

C. papaya belongs to family Caricaceae. Previously papaya seeds and fruits are reported to have powerful inhibitory compound benzyl isothiocyanate (BITC). This compound has fungicidal, bactericidal and insecticidal activity. Two closely related allelocompounds (allyl isothiocyanate, phenethyl isothiocyanate) are known to be germination inhibitors (Wolf et al., 1984). The specific objectives of current study were:

1. Assess herbicidal potential of C. papaya by 'plant leaf powder bioassay' against seeds and pre-germinated seeds of selected test species on filter paper and soil;

2. Assess herbicidal potential of C. papaya against seeds and pre-germinated seeds of selected test species through 'aqueous extract bioassay' on filter paper and soil.

\section{Material and Methods}

\subsection{Collection of plant material and procurement of seeds of test species}

Mature, healthy leaf material of C. papaya was collected from roadside of Rawalpindi District $\left(73^{\circ} 02^{\prime} \mathrm{E}\right.$ longitude; $33^{\circ} 36^{\prime} \mathrm{N}$ latitude), Pakistan. Collected leaves were thoroughly washed under running tap water to remove any dust particles and shade dried till complete drying (six days in our case). The dried leaf material was then ground to fine powder. Seeds of selected test species viz. A. fatua, R. dentatus, T. aestivum (Wafaq 2001), H. annuиs (K.S.E 7777) and Z. mays (Islamabad Gold 2010) were procured from Department of Crop Science, National Agriculture Research Centre, Islamabad, Pakistan. 1\% sodium hypochloride $(\mathrm{NaClO})$ solution was used to surface sterilize seeds of test species.

\subsection{Aqueous extract bioassay}

Dried leaf powder $(10 \mathrm{~g})$ of $C$. papaya was soaked in $200 \mathrm{ml}$ distilled water in round bottom flask and agitated on orbital shaker $(150 \mathrm{rpm})$ for twenty four hours at room temperature $\left(25^{\circ} \mathrm{C}\right)$. The extract was filtered through Whatman filter paper No. 1. Screening for seeds and pre-germinated seeds was carried out on filter paper and soil. For screening on filter paper, $5 \mathrm{ml}$ leaf extract was poured into petri dishes underlined with filter paper. Ten surface sterilized seeds of each test species were placed in petri dishes. The petri dishes were then sealed with parafilm, covered with aluminum foil and incubated 
Table 1. Common allelopathic compounds and representing plants.

\begin{tabular}{|c|c|}
\hline Plant species & Isolated Allelochemicals \\
\hline Acroptilon repens (L.) DC. & $\begin{array}{l}\text { Cnicin (Sesquiterpene lactone) and 7,8-benzoflavone } \\
\text { (Flavone) }\end{array}$ \\
\hline Alliaria petiolata (Bieb.) Cavara and Grande & Alliarinoside (Glucoside) \\
\hline Artemisia tridentate ssp. tridentata & Methyl jasmonate (Volatile organic compound) \\
\hline Berberis thunbergii DC. & Berberine (Alkaloid) \\
\hline Carduus nutans L. and C. acanthoides L. & Aplotaxene (Essential oil) \\
\hline Celastrus orbiculatus Thunb. & Celastrol (Terpenoid) \\
\hline Centaurea biebersteinii DC. & (-)-Catechin (Flavonoid) \\
\hline Centaurea diffusa Lam. & $\begin{array}{l}\text { 8-Hydroxyquinoline (Alkaloid), Caryophyllene oxide } \\
\text { (Sesquiterpene), Linoleic acid (Fatty acid) }\end{array}$ \\
\hline Centaurea maculosa Lam. & $( \pm)$ - Catechin (Phenol) \\
\hline Centrostachys aquatica (R.Br.) Moq. & Loliolide (Terpene) \\
\hline Chenopodium ambrosioides (L.) Mosyakin \& Clemants & $\begin{array}{l}\alpha \text {-terpinene (Terpene), limonene (Terpene), } \\
\text { ascaridole(Terpene) }\end{array}$ \\
\hline Cytisusscoparius (L.) Link & Sparteine (Alkaloid) \\
\hline Elaeagnus angustifolia L. & Harman (Amine) \\
\hline Euphorbia esula L. & Esulatin (Terpenoid) \\
\hline Geranium carolinianum L. & Ethyl gallate (Phenol) \\
\hline Hieracium pilosella $\mathrm{L}$. & Umbelliferone (Coumarin) \\
\hline Hypericum perforatum L. & Hypericin (Anthraquinone) \\
\hline Hyptis suaveolens (L.) Poit. & Suaveolic Acid (Terpenoid) \\
\hline Imperata cylindrica (L.) P.Beauv. & Tabanone, Hexadecahydro-1-azachrysen-8-yl ester (Alkaloid) \\
\hline Linaria dalmatica (L.) P. Mill. & Linarioside (Glocoside) \\
\hline Lythrum salicaria $\mathrm{L}$. & Gallic acid (Phenolic acid) \\
\hline Melaleuca quinquenervia (Cav.) Blake & Nerolidol (Sesquiterpene) \\
\hline Mikania micrantha H.B.K. & $\begin{array}{l}\text { Deoxymikanolide (Sesquiterpenoids), dihydromikanolide } \\
\text { (Sesquiterpenes), 2,3-epoxy-1-hydroxy-4,9- } \\
\text { germacradiene-12,8:15,6-diolide (Sesquiterpenoid) }\end{array}$ \\
\hline Pastinaca sativa L. & Xanthotoxin (Coumarin) \\
\hline Phragmites australis & Gallic acid (Phenolic acid) \\
\hline Polygonum cuspidatum Sieb. And Zucc. & Piceid (Glucoside) \\
\hline Pueraria montana (Lour.) Merr. & Biochanin-A (Isoflavone) \\
\hline Rhamnus cathartica $\mathrm{L}$. & Emodin (Resin) \\
\hline Sapium sebiferum (L.) Roxb. & Xanthoxylin (Phenol) \\
\hline
\end{tabular}

in growth chamber at room temperature. Germination percentage $(\%)$, plumule and radicle length $(\mathrm{cm})$ of test species were recorded after fifteen days with reference to control. For screening on soil, $15 \mathrm{ml}$ leaf extract was poured into petri dishes underlined with $25 \mathrm{~g}$ soil.

\subsection{Plant leaf powder bioassay}

C. papaya leaf powder $(0.25 \mathrm{~g})$ was added to filter paper in petri dish along with $5 \mathrm{ml}$ distilled water. In each petri dish, ten surface sterilized seeds of test species were placed. The petri dishes were sealed with parafilm, covered with aluminum foil and incubated in growth chamber at room temperature. Germination percentage (\%), plumule and radicle length $(\mathrm{cm})$ were recorded after fifteen days with reference to control. For screening on soil, C. papaya leaf powder $(0.25 \mathrm{~g})$ was added to $25 \mathrm{~g}$ soil along with $15 \mathrm{ml}$ distilled water in petri dish. $15 \mathrm{ml}$ distilled water was used in petri dishes as control without adding leaf powder on soil and parameters were studied for bioassay.

\subsection{Statistical analysis}

STATISTIX 9 was used for data interpretation and mean values of studied parameters were separated by Fisher's protected LSD test (Steel and Torrie, 1997).

\section{Results}

The data analysis indicated that application of plant aqueous extract and dry powdered material of C. papaya leaves (next mentioned as 'screening material') significantly reduced germination percentage (\%) of all test species viz. A. fatua, $R$. dentatus, $H$. annuus, Z. mays and T. aestivum Although germination inhibition (\%) was observed for all test species by dry powdered form and aqueous extract of screening material on filter paper and soil, most significant inhibition in germination percentage (\%) was observed by its aqueous extract applied on filter paper. Among test species, germination of $A$. fatua seeds was most affected by $C$. papaya plant material in each form. For other test 
species, reduction in germination was observed in $R$. dentatus, followed by $Z$. mays and $H$. annuus with almost similar inhibitory effects. However, germination of T. aestivum was least affected with the application of both aqueous extract and dry powdered material (Table 2). Likewise, the effects of C. papaya on seed germination, most of the radicle growth inhibition was also observed in the seedlings of $A$. fatua on filter paper medium. The radicle length was significantly reduced by C. papaya, both in aqueous $(80 \%)$ and dry powdered form (89\%) (Table 3-4). On the other hand, plumule growth was also reduced under the influence of aqueous extract (57-73\%) and powdered material (59-77\%) (Table 5-6). The inhibitory effects on other test species were in the sequence of $H$. annuus, followed by Z. mays, $R$. dentatus and T. aestivum being the least affected test species among all. In a comparison between the effects on seed and pre-germinated seeds, more inhibition was found in pre-germinated seeds of different test species under the influence of aqueous extract and powdered plant material. $R$. dentatus was mostly affected by aqueous extract of C. papaya compared to the application of plant material in powdered form. The inhibitory effects were found in

Table 2. Germination percentage of seeds of test species in aqueous extract and leaf dry powder of C. papaya in filter papers and soil.

\begin{tabular}{|c|c|c|c|c|c|c|}
\hline \multirow{2}{*}{\multicolumn{2}{|c|}{ Treatments }} & \multicolumn{5}{|c|}{ Test Species } \\
\hline & & \multirow{2}{*}{$\frac{\text { A. fatua }}{20^{\mathrm{b}}}$} & \multirow{2}{*}{$\frac{\text { H. annuus }}{59^{\mathrm{b}}}$} & \multirow{2}{*}{$\frac{\text { R. dentatus }}{26^{\mathrm{b}}}$} & \multirow{2}{*}{$\frac{\text { T. aestivum }}{95^{\mathrm{a}}}$} & \multirow{2}{*}{$\frac{\text { Z. mays }}{48^{\mathrm{b}}}$} \\
\hline Aqueous extract & Filter Paper & & & & & \\
\hline & Control & $86^{\mathrm{a}}$ & $100^{\mathrm{a}}$ & $80^{\mathrm{a}}$ & $99^{\mathrm{a}}$ & $92^{\mathrm{a}}$ \\
\hline & ${ }^{1} \mathrm{LSD}$ & 14.197 & 17.604 & 14.541 & 17.902 & 19.778 \\
\hline & ${ }^{2} \mathrm{~F}$-value & $42 *$ & $14.67 *$ & $43.98^{*}$ & $12.13 *$ & $13.52 *$ \\
\hline & Soil & $29^{\mathrm{b}}$ & $50^{\mathrm{b}}$ & $21^{\mathrm{b}}$ & $91^{\mathrm{a}}$ & $56^{\mathrm{b}}$ \\
\hline & Control & $90^{\mathrm{a}}$ & $96^{\mathrm{a}}$ & $89^{a}$ & $94^{\mathrm{a}}$ & $93^{\mathrm{a}}$ \\
\hline & ${ }^{1} \mathrm{LSD}$ & 17.510 & 14.744 & 16.580 & 13.554 & 14.08 \\
\hline & ${ }^{2} \mathrm{~F}$-value & $22.36^{*}$ & $21.04 *$ & $51.38 *$ & $13.63^{*}$ & $38.81^{*}$ \\
\hline \multirow[t]{8}{*}{ Dry powder } & Filter Paper & $36^{\mathrm{b}}$ & $56^{\mathrm{b}}$ & $55^{\mathrm{b}}$ & $96^{\mathrm{a}}$ & $40^{\mathrm{b}}$ \\
\hline & Control & $95^{\mathrm{a}}$ & $97^{\mathrm{a}}$ & $86^{\mathrm{a}}$ & $98^{\mathrm{a}}$ & $82^{\mathrm{a}}$ \\
\hline & ${ }^{1} \mathrm{LSD}$ & 17.66 & 16.974 & 17.529 & 14.722 & 16.974 \\
\hline & ${ }^{2} \mathrm{~F}$-value & $26.08 *$ & $13.78 *$ & $21.73 *$ & $27.64 *$ & $13.29 *$ \\
\hline & Soil & $49^{b}$ & $37^{\mathrm{b}}$ & $32^{\mathrm{b}}$ & $93^{\mathrm{a}}$ & $29^{b}$ \\
\hline & Control & $99^{\mathrm{a}}$ & $99^{\mathrm{a}}$ & $89^{\mathrm{a}}$ & $96^{\mathrm{a}}$ & $84^{\mathrm{a}}$ \\
\hline & ${ }^{1} \mathrm{LSD}$ & 18.890 & 18.60 & 17.67 & 18.608 & 17.435 \\
\hline & ${ }^{2} \mathrm{~F}$-value & $17.19 *$ & $33.19 *$ & $32.71 *$ & $10.89 *$ & $21.97 *$ \\
\hline
\end{tabular}

Note: ${ }^{1}$ Means followed by different letters within one column differ significantly at $(P<5 \%) ;{ }^{2 *}=$ Significant at $(P<1 \%)$.

Table 3. Radicle length $(\mathrm{cm})$ of seeds and pre-germinated seeds of test species as suppressed by aqueous extract of $C$. papaya in filter papers and soil.

\begin{tabular}{|c|c|c|c|c|c|c|}
\hline \multirow{2}{*}{\multicolumn{2}{|c|}{ Treatments }} & \multicolumn{5}{|c|}{ Test species } \\
\hline & & A. fatua & H. annuus & R. dentatus & T. aestivum & Z. mays \\
\hline \multirow[t]{8}{*}{ Seeds } & Filter Paper & $4.32^{\mathrm{b}}$ & $5.11^{\mathrm{b}}$ & $6.21^{\mathrm{b}}$ & $12.6^{\mathrm{a}}$ & $4.12^{b}$ \\
\hline & Control & $11.2^{\mathrm{a}}$ & $9.48^{\mathrm{a}}$ & $8.03^{\mathrm{a}}$ & $13.15^{\mathrm{a}}$ & $10.3^{\mathrm{a}}$ \\
\hline & ${ }^{1} \mathrm{LSD}$ & 1.0971 & 1.0360 & 1.0027 & 3.0879 & 0.6573 \\
\hline & ${ }^{2} \mathrm{~F}$-value & $126.95^{*}$ & $128.48^{*}$ & $92.48^{*}$ & $18.78^{*}$ & $309.12 *$ \\
\hline & Soil & $2.48^{b}$ & $2.48^{b}$ & $5.46^{\mathrm{b}}$ & $11.2^{\mathrm{a}}$ & $3.29^{\mathrm{b}}$ \\
\hline & Control & $10.45^{\mathrm{a}}$ & $8.39^{\mathrm{a}}$ & $7.17^{\mathrm{a}}$ & $11.4^{\mathrm{a}}$ & $8.45^{\mathrm{a}}$ \\
\hline & ${ }^{1} \mathrm{LSD}$ & 1.1918 & 1.0634 & 0.8063 & 1.8106 & 1.0912 \\
\hline & ${ }^{2} \mathrm{~F}$-value & $98.97 *$ & $74.89 *$ & $126.70 *$ & $52.46^{*}$ & $83.01 *$ \\
\hline \multirow{8}{*}{$\begin{array}{l}\text { Pre- } \\
\text { germinated } \\
\text { seeds }\end{array}$} & Filter Paper & $2.09^{\mathrm{b}}$ & $3.48^{\mathrm{b}}$ & $1.24^{\mathrm{b}}$ & $14.9^{\mathrm{a}}$ & $2.37^{\mathrm{b}}$ \\
\hline & Control & $10.2^{\mathrm{a}}$ & $10.1^{\mathrm{a}}$ & $5.33^{\mathrm{a}}$ & $16.2^{\mathrm{a}}$ & $7.27^{\mathrm{a}}$ \\
\hline & ${ }^{1} \mathrm{LSD}$ & 0.4135 & 2.0401 & 0.7263 & 3.4976 & 1.5567 \\
\hline & ${ }^{2} \mathrm{~F}$-value & $740.81^{*}$ & $30.76^{*}$ & $64.28 *$ & $18.78^{*}$ & $22.96^{*}$ \\
\hline & Soil & $2.28^{\mathrm{b}}$ & $2.17^{\mathrm{b}}$ & $3.07^{\mathrm{b}}$ & $12.1^{\mathrm{a}}$ & $2.12^{\mathrm{b}}$ \\
\hline & Control & $9.11^{\mathrm{a}}$ & $8.49^{\mathrm{a}}$ & $4.11^{\mathrm{a}}$ & $13.4^{\mathrm{a}}$ & $6.42^{\mathrm{a}}$ \\
\hline & ${ }^{1} \mathrm{LSD}$ & 0.8095 & 1.9073 & 0.9043 & 2.5542 & 1.2569 \\
\hline & ${ }^{2} \mathrm{~F}$-value & $206.09 *$ & $27.90 *$ & $20.88^{*}$ & $23.58 *$ & $39.32 *$ \\
\hline
\end{tabular}

Note: ${ }^{1}$ Means followed by different letters within one column differ significantly at $(P<5 \%) ;{ }^{2 *}=$ Significant at $(P<1 \%)$. 
Table 4. Radicle length $(\mathrm{cm})$ of seeds and pre-germinated seeds of test species as suppressed by leaf dry powder of $C$. papaya in filter papers and soil.

\begin{tabular}{|c|c|c|c|c|c|c|}
\hline \multirow{2}{*}{\multicolumn{2}{|c|}{ Treatments }} & \multicolumn{5}{|c|}{ Test species } \\
\hline & & A. fatua & H. annuus & R. dentatus & T. aestivum & Z. mays \\
\hline \multirow[t]{8}{*}{ Seeds } & Filter Paper & $1.01^{\mathrm{b}}$ & $4.25^{\mathrm{b}}$ & $5.49^{\mathrm{b}}$ & $13.1^{\mathrm{a}}$ & $3.23^{b}$ \\
\hline & Control & $9.34^{\mathrm{a}}$ & $8.49^{\mathrm{a}}$ & $7.07^{\mathrm{a}}$ & $14.3^{\mathrm{a}}$ & $9.01^{\mathrm{a}}$ \\
\hline & ${ }^{1} \mathrm{LSD}$ & 0.5692 & 1.4388 & 1.203 & 3.2679 & 0.6136 \\
\hline & ${ }^{2} \mathrm{~F}$-value & $485.76^{*}$ & $56.00^{*}$ & $56.71 *$ & $22.22 *$ & $279.70^{*}$ \\
\hline & Soil & $2.47^{\mathrm{b}}$ & $6.06^{\mathrm{b}}$ & $6.45^{\mathrm{b}}$ & $10.3^{\mathrm{a}}$ & $4.31^{\mathrm{b}}$ \\
\hline & Control & $8.05^{\mathrm{a}}$ & $9.30^{\mathrm{a}}$ & $8.46^{\mathrm{a}}$ & $9.49^{\mathrm{a}}$ & $11.1^{\mathrm{a}}$ \\
\hline & ${ }^{1} \mathrm{LSD}$ & 0.999 & 1.0662 & 1.320 & 1.540 & 1.1125 \\
\hline & ${ }^{2} \mathrm{~F}$-value & $66.33^{*}$ & $95.32 *$ & $56.72 *$ & $45.47 *$ & $111.84^{*}$ \\
\hline \multirow{8}{*}{$\begin{array}{l}\text { Pre-germinated } \\
\text { seeds }\end{array}$} & Filter Paper & $2.07^{\mathrm{b}}$ & $2.08^{\mathrm{b}}$ & $2.10^{\mathrm{b}}$ & $1.9^{\mathrm{a}}$ & $3.30^{\mathrm{b}}$ \\
\hline & Control & $12.3^{\mathrm{a}}$ & $9.45^{\mathrm{a}}$ & $6.07^{\mathrm{a}}$ & $13.4^{\mathrm{a}}$ & $8.07^{\mathrm{a}}$ \\
\hline & ${ }^{1} \mathrm{LSD}$ & 0.9870 & 1.1352 & 0.9713 & 2.4770 & 1.4150 \\
\hline & ${ }^{2} \mathrm{~F}$-value & $209.06^{*}$ & $110.25^{*}$ & $49.22 *$ & $18.01 *$ & $41.84 *$ \\
\hline & Soil & $3.38^{\mathrm{b}}$ & $1.17^{\mathrm{b}}$ & $2.17^{b}$ & $11.8^{\mathrm{a}}$ & $1.49^{b}$ \\
\hline & Control & $10.05^{\mathrm{a}}$ & $8.00^{\mathrm{a}}$ & $5.21^{\mathrm{a}}$ & $13.4^{\mathrm{a}}$ & $8.14^{\mathrm{a}}$ \\
\hline & ${ }^{1} \mathrm{LSD}$ & 0.8763 & 1.2929 & 0.8953 & 3.1490 & 1.7841 \\
\hline & ${ }^{2} \mathrm{~F}$-value & $129.81^{*}$ & $66.06^{*}$ & $50.17 *$ & $22.59 *$ & $34.17 *$ \\
\hline
\end{tabular}

Note: ${ }^{1}$ Means followed by different letters within one column differ significantly at $(P<5 \%) ;{ }^{2 *}=$ Significant at $(P<1 \%)$.

Table 5. Plumule length $(\mathrm{cm})$ of seeds and pre-germinated seeds of test species as suppressed by aqueous extract of C.papaya in filter papers and soil.

\begin{tabular}{|c|c|c|c|c|c|c|}
\hline \multirow{2}{*}{\multicolumn{2}{|c|}{ Treatments }} & \multicolumn{5}{|c|}{ Test species } \\
\hline & & \multirow{2}{*}{$\frac{\text { A. fatua }}{4.34^{\mathrm{b}}}$} & \multirow{2}{*}{$\frac{\text { H. annuus }}{2.22^{\mathrm{b}}}$} & \multirow{2}{*}{$\frac{\text { R. dentatus }}{2.12^{\mathrm{b}}}$} & \multirow{2}{*}{$\frac{\text { T. aestivum }}{7.05^{\mathrm{a}}}$} & \multirow{2}{*}{$\frac{\text { Z. mays }}{4.22^{\mathrm{b}}}$} \\
\hline Seeds & Filter Paper & & & & & \\
\hline & Control & $10.15^{\mathrm{a}}$ & $6.39^{\mathrm{a}}$ & $8.06^{\mathrm{a}}$ & $8.16^{\mathrm{a}}$ & $8.45^{\mathrm{a}}$ \\
\hline & ${ }^{1} \mathrm{LSD}$ & 0.6647 & 0.9468 & 0.6294 & 1.3210 & 1.0678 \\
\hline & ${ }^{2} \mathrm{~F}$-value & $238.96^{*}$ & $37.04 *$ & $197.36^{*}$ & $45.63^{*}$ & $97.24^{*}$ \\
\hline & Soil & $2.34^{\mathrm{b}}$ & $2.00^{\mathrm{b}}$ & $1.69^{\mathrm{b}}$ & $7.32^{\mathrm{a}}$ & $3.09^{\mathrm{b}}$ \\
\hline & Control & $7.40^{\mathrm{a}}$ & $8.37^{\mathrm{a}}$ & $7.45^{\mathrm{a}}$ & $8.49^{a}$ & $7.00^{\mathrm{a}}$ \\
\hline & ${ }^{1} \mathrm{LSD}$ & 0.8978 & 1.0434 & 1.0019 & 2.3502 & 0.7620 \\
\hline & ${ }^{2} \mathrm{~F}$-value & $71.85^{*}$ & $73.9 *$ & $87.41^{*}$ & $9.64 *$ & $101.44 *$ \\
\hline \multirow{8}{*}{$\begin{array}{l}\text { Pre-germinated } \\
\text { seeds }\end{array}$} & Filter Paper & $3.01^{\mathrm{b}}$ & $4.49^{\mathrm{b}}$ & $2.12^{\mathrm{b}}$ & $10.1^{\mathrm{a}}$ & $4.01^{b}$ \\
\hline & Control & $9.08^{\mathrm{a}}$ & $10.05^{\mathrm{a}}$ & $9.20^{\mathrm{a}}$ & $11.8^{\mathrm{a}}$ & $11.45^{\mathrm{a}}$ \\
\hline & ${ }^{1} \mathrm{LSD}$ & 0.3036 & 1.5861 & 0.8317 & 2.5454 & 1.7866 \\
\hline & ${ }^{2} \mathrm{~F}$-value & $825.69 *$ & $47.89 *$ & $152.73^{*}$ & $14.38 *$ & $80.15^{*}$ \\
\hline & Soil & $2.30^{\mathrm{b}}$ & $4.16^{\mathrm{b}}$ & $2.01^{\mathrm{b}}$ & $8.96^{\mathrm{a}}$ & $7.13^{b}$ \\
\hline & Control & $8.48^{\mathrm{a}}$ & $9.08^{\mathrm{a}}$ & $7.44^{\mathrm{a}}$ & $10.1^{\mathrm{a}}$ & $13.2^{\mathrm{a}}$ \\
\hline & ${ }^{1} \mathrm{LSD}$ & 1.2227 & 1.2301 & 0.9318 & 2.3413 & 1.2891 \\
\hline & ${ }^{2} \mathrm{~F}$-value & $54.40^{*}$ & $56.75^{*}$ & $69.05^{*}$ & $20.36^{*}$ & $88.02 *$ \\
\hline
\end{tabular}

Note: ${ }^{1}$ Means followed by different letters within one column differ significantly at $(P<5 \%) ;{ }^{2 *}=$ Significant at $(P<1 \%)$.

the range of $73-77 \%$ plumule and $23-77 \%$ radicle, both in seeds and pre-germinated seeds (Table 2-5). The inhibitory effects of C. papaya on $Z$. mays and H. annuus were somehow like each other. $82 \%$ radicle growth inhibition was found in soil experiment of $Z$. mays with powdered plant material while in case of $H$. annuus, these effects were up to $85 \%$ (Table 2-3). On the other hand, there was up to $67 \%$ radicle growth inhibition in $Z$. mays with plant aqueous extract while $76 \%$ plumule growth inhibition in $H$. annuus with the same extract. Apart from the effects on above mentioned four test species, T. aestivum was found to be the least affected test species. Aqueous extract of C. papaya inhibited $2-10 \%$ radicle and $11-14 \%$ plumule growth in both seed and seedling stages with soil and filter paper experiments (Table $2 \& 4$ ). C. papaya in dry powdered form variably affected the growth of this test species. Maximum inhibition (86\%) was found in radicle growth of pre-germinated seedlings while $9 \%$ stimulation was also observed in radicle growth of seeds of this plant species (Table 4 and 6). 
Table 6. Plumule length $(\mathrm{cm})$ of seeds and pre-germinated seeds of test species as suppressed by leaf dry powder of C.papaya in filter papers and soil.

\begin{tabular}{|c|c|c|c|c|c|c|}
\hline \multirow{2}{*}{\multicolumn{2}{|c|}{ Treatments }} & \multicolumn{5}{|c|}{ Test species } \\
\hline & & A. fatua & H. annuus & R. dentatus & T. aestivum & Z. mays \\
\hline \multirow[t]{8}{*}{ Seeds } & Filter Paper & $2.47^{\mathrm{b}}$ & $2.23^{\mathrm{b}}$ & $0.02^{\mathrm{b}}$ & $7.65^{\mathrm{a}}$ & $4.09^{\mathrm{b}}$ \\
\hline & Control & $8.49^{a}$ & $5.42^{\mathrm{a}}$ & $7.96^{\mathrm{a}}$ & $9.48^{\mathrm{a}}$ & $7.46^{\mathrm{a}}$ \\
\hline & ${ }^{1} \mathrm{LSD}$ & 1.2971 & 0.8718 & 0.0182 & 2.7846 & 0.9640 \\
\hline & ${ }^{2} \mathrm{~F}$-value & $46.74 *$ & $55.81 *$ & $14.11 *$ & $6.44 *$ & $57.31 *$ \\
\hline & Soil & $3.00^{\mathrm{b}}$ & $2.12^{\mathrm{b}}$ & $2.12^{\mathrm{b}}$ & $11.25^{\mathrm{a}}$ & $5.45^{\mathrm{b}}$ \\
\hline & Control & $7.44^{\mathrm{a}}$ & $6.40^{\mathrm{a}}$ & $9.07^{\mathrm{a}}$ & $12.15^{\mathrm{a}}$ & $8.07^{\mathrm{a}}$ \\
\hline & ${ }^{1} \mathrm{LSD}$ & 1.4091 & 1.1428 & 0.7297 & 2.2332 & 0.9679 \\
\hline & ${ }^{2} \mathrm{~F}$-value & $26.83^{*}$ & $29.95^{*}$ & $221.41 *$ & $28.95^{*}$ & $83.03 *$ \\
\hline \multirow{8}{*}{$\begin{array}{l}\text { Pre-germinated } \\
\text { seeds }\end{array}$} & Filter Paper & $2.13 b$ & $4.09^{\mathrm{b}}$ & $2.00^{\mathrm{b}}$ & $9.35^{\mathrm{a}}$ & $5.30^{\mathrm{b}}$ \\
\hline & Control & $9.45^{\mathrm{a}}$ & $9.45^{\mathrm{a}}$ & $8.12^{\mathrm{a}}$ & $9.35^{\mathrm{a}}$ & $11.4^{\mathrm{a}}$ \\
\hline & ${ }^{1} \mathrm{LSD}$ & 0.9834 & 1.2510 & 0.6111 & 1.8694 & 2.1870 \\
\hline & ${ }^{2} \mathrm{~F}$-value & $124.1^{*}$ & $57.71 *$ & $216.40 *$ & $17.04 *$ & $37.65^{*}$ \\
\hline & Soil & $3.31^{\mathrm{b}}$ & $3.25^{\mathrm{b}}$ & $2.13^{b}$ & $7.01^{\mathrm{a}}$ & $2.14^{\mathrm{b}}$ \\
\hline & Control & $8.07^{\mathrm{a}}$ & $8.06^{\mathrm{a}}$ & $7.10^{\mathrm{a}}$ & $8.45^{\mathrm{a}}$ & $10.05^{\mathrm{a}}$ \\
\hline & ${ }^{1} \mathrm{LSD}$ & 1.0523 & 1.1964 & 0.9823 & 2.1252 & 0.8164 \\
\hline & ${ }^{2} \mathrm{~F}$-value & $48.12 *$ & $66.81 *$ & $66.95 *$ & $70.87 *$ & $280.93 *$ \\
\hline
\end{tabular}

Note: ${ }^{1}$ Means followed by different letters within one column differ significantly at $(P<5 \%) ;{ }^{2 *}=$ Significant at $(P<1 \%)$.

\section{Discussion}

C. papaya belongs to a small family Caricaceae. It is succulent plant growing in tropical countries and sub-tropical regions of the world. Allelochemicals are released by different parts of the plant and leaves are most active in this respect (Rehman et al., 2014). The plant is reported to possess organic acids, alkaloids, tannins and flavonoids which hamper growth of adjacent plants (Canini et al., 2007). C. papaya is known to influence the growth of plants in its vicinity by the release of the allelochemicals during the decomposition of its litter (Christobel et al., 2017). Allelopathic potential of C. papaya leaves for pest management is reported earlier. Crude aqueous leaf extracts of C. papaya on susceptibility of fourth instar larvae of Spodoptera litura showed a significant total percent mortality (Deshmukhe et al., 2009). Therefore, allelopathic effect of leaves of C. papaya was evaluated for its aqueous extract as well as dry powder against five test species i.e., T. aestivum, A. fatua, H. annuus, Z. mays and $R$. dentatus. For each test species, screening plant material was applied on filter paper and soil separately in both aqueous extract and powdered form that variably reduced the germination and growth of test plants.

In our experiments, $C$. papaya affected germination of all test species. Similar to our results, inhibition of seed germination was observed in Sinapis arvensis by Glaucium species (Ghorbanli et al., 2011), in sorghum, sesame, sunflower and corn by mungbean (Lertmongkol et al., 2011), in chive and soybean by ginger (Han et al., 2008). Reigosa et al. (1999) proposed that allelochemicals affect physiological functions during germination thus hindering the process. It was reported that, seed characteristics such as seed coat permeability and seed size influence effects of allelochemicals in seeds and interference of the allelochemical compounds vary accordingly (Marianne et al., 2000). Similar results were proposed by Gherardi and Valio (1976) and Chow and Lin (1991) in bioassays with lettuce seeds and by Reyes et al. (1980) with cucumber seeds. Chow and Lin (1991) used extracts of sarcotesta and seeds of papaya, which showed presence of germination and growth inhibitors in the sarcotesta, halting the germination of lettuce seeds, which had also observed earlier by Gherardi and Valio (1976). They state that such inhibitors are phenolic in nature. In our study, most significant inhibition effects were found on A. fatua as compared to other test species. Similar results were reported by Turk and Tawaha (2003) showing negative germination effects of aqueous extract of Brassica nigra on A. fatua. A. fatua was also found to be a susceptible species against $P$. hysterophorus (Batish et al., 2002; Marwat et al., 2008; Anwar et al., 2019). These observations have indicated that this test plant species is susceptible to number of allelopathic plants (Anwar et al., 2017a, 2018c).

Radicle and plumule length inhibition was obsered in test species. Kato-Noguchi (2011) also observed epicotyl and hypocotyl inhibition by the allelopathic extracts of different plants. Similar results were reported by Oudhi (2001), Anwar et al. (2018d) and Maharjan et al. (2007). Allelopathic assessment of $C$. papaya leaves, in our study, indicated that radicle length was more affected than plumule length. These findings support root sensitivity to allelochemicals compared to shoot. Relative greater root sensitivity may be explained by the fact, that after the seed germination, roots are first to be in contact with toxic compounds (Qureshi et al., 2014). Similar results were proposed by Fujii and Aziz (2005) while examining 
allelopathic effect of fourteen different plant species extracts on growth of lettuce seeds. The inhibitory actions under influence of allelochemicals are possibly due to reduction in cell division and cell size (Einhellig, 1996). The aqueous extract of C. papaya leaves, however, showed non-significant effect on wheat seeds germination, radicle and plumule growth. Similar results were reported by Tanveer et al. (2007) and Anwar et al. (2017b), where E. helioscopia was found to be non-toxic against wheat growth. Researches have suggested that a specific dose of these chemicals may cause growth stimulation (Pelinganga and Mashela, 2012). Abu El-Soud (2001) demonstrated growth stimulation in pea crop by Acacia nilotica leaf residue. Christobel et al. (2017) stated that C. papaya aqueous leaf extract can be applied for growth improvement of Phaseolus aureus. Stimulation is suggested usually due to presence of growth promoting substances along with inhibitory substances (Yamada et al., 2010; Anwar et al., 2018a, b).

The results of the present work clearly indicate that $C$. papaya is a candidate of consideration for weed management in wheat crop. It was reported elsewhere that C. The papaya leaf extract is source of phenolic substances, acknowledged as the basis of allopathic properties of the plant species (Golisz et al., 2007). Understanding the mechanisms may contribute to the use of plant compounds as natural herbicide, which will help to limit use of synthetic herbicides.

\section{Conclusions}

Biochemical evaluation of plants plays an important role in the sustainability and improvement of agro-ecosystems. Results of this study indicated that A. fatua was more susceptible towards the allelopathic effects of $C$. papaya. Donor plant in both extract and powdered form greatly reduced the germination and growth of $A$. fatua. Moreover, these inhibitory effects were greater for extract than powdered form due to the presence of water soluble allelochemicals. The growth of $R$. dentatus was less inhibited as compared to A. fatua. While the inhibitory effects on Z. mays and $H$. annuus were somehow like other test species. Wheat was observed to be least affected test species against $C$. papaya. The aqueous extract of $C$. papaya leaves is locally available in sufficient quantities; biodegradable, economic to the farmer hence can be effectively utilized for weed management in wheat crop. It might be suggested that to exploit this plant as natural herbicide, its allelochemicals and their effects must be studied in detail with more advanced techniques. On the other hand, it might be suggested that C. papaya should not be introduced in intercropping system due to its negative effects on food crops.

\section{References}

ABU EL-SOUD, W.M., 2001. Allelopathic potential of Acacia nilotica tree leaves on the metabolic changes of Pea (Pisum sativum). Egypt: Faculty of Science, Cairo University, 84 p. M. Sc. Thesis in Botany.
ALAGESABOOPATHI, C., 2010. Allelopathic effects of Centella asiatica aqueous extracts on pearl millet (Pennisetum typhoides L.) and cowpea (Vigna unguiculata Walp.). Pakistan Journal of Weed Science Research, vol. 16, no. 1, pp. 67-71.

ANWAR, T., ILYAS, N., QURESHI, R. and MALIK, M.A., 2019. Allelopathic potential of Carica papaya against selected weeds of wheat crop. Pakistan Journal of Botany, vol. 51, no. 1, pp. 1-37. http://dx.doi.org/10.30848/PJB2019-1(37).

ANWAR, T., KHALID, S., SAEED, M., MAZHAR, R., QURESHI, H. and RASHID, M., 2016. Allelopathic interference of leaf powder and aqueous extracts of hostile weed: Parthenium hysterophorus (Astraceae). Science International, vol. 4, no. 3, pp. 86-93. http://dx.doi.org/10.17311/sciintl.2016.86.93.

ANWAR, T., KHALID, S., MAZHAR, R., QURESHI, H. and RASHID, M., 2017a. Herbicidal potential of selected species to overcome weed infestation in Triticum aestivum, Zea mays and Helianthus annuus. Pakistan Journal of Weed Science Research, vol. 23, no. 1, pp. 49-63.

ANWAR, T., KHALID, S., PANNI, M.K., QURESHI, H. and RASHID, M., 2017b. Allelopathic effect of Euphorbia helioscopia on Avena fatua, Rumex dentatus, Helianthus annuus, Zea mays and Triticum aestivum. Pakistan Journal of Weed Science Research, vol. 23, no. 2, pp. 165-177.

ANWAR, T., ILYAS, N., QURESHI, R., MUNAZIR, M., KHAN, A.M., ANSARI, L., RAHIM, B.Z., ANSARI, K.A. and PANNI, M.K., 2018a. Allelopathic activity of solvent extracts of Rhazya stricta Decne. against selected weeds of wheat crop. Applied Ecology and Environmental Research, vol. 16, no. 5, pp. 5405-5421. http://dx.doi.org/10.15666/aeer/1605_54055421.

ANWAR, T., ILYAS, N., QURESHI, R., MAQSOOD, M., MUNAZIR, M., ANWAR, P., RAHIM, B.Z., ANSARI, K.A. and PANNI, M.K., 2018b. Allelopathic potential of Lantana camara against selected weeds of wheat crop. Applied Ecology and Environmental Research, vol. 16, no. 5, pp. 6741-6760. http:// dx.doi.org/10.15666/aeer/1605_67416760.

ANWAR, T., PANNI, M.K., KHALID, S. and QURESHI, H., $2018 \mathrm{c}$. Appraisal of allelopathic potential of curly dock (Rumex dentatus L.) as a natural weed management source. Pakistan Journal of Weed Science Research, vol. 24, no. 3, pp. 231-239. http://dx.doi.org/10.28941/24-3(2018)-5.

ANWAR, T., PANNI, M.K., KHALID, S. and QURESHI, H., 2018d. Allelopathic management of noxious weeds in Helianthus annuus, Zea mays and Triticum aestivum by selected plants. Pakistan Journal of Weed Science Research, vol. 24, no. 3, pp. 257-265. http://dx.doi.org/10.28941/24-3(2018)-7.

BATISH, D.R., LAVANYA, K., PAL SINGH, H. and KOHLI, R.K., 2007. Root-mediated allelopathic interference of nettleleaved goosefoot (Chenopodium murale) on wheat (Triticum aestivum). Journal Agronomy \& Crop Science, vol. 193, no. 1, pp. 37-44. http://dx.doi.org/10.1111/j.1439-037X.2006.00243.x.

BATISH, D.R., SINGH, H.P., KOHLI, R.K., SAXENA, D.B. and KAUR, S., 2002. Allelopathic effect of parthenin against two weedy species Avena fatua and Bidens pilosa. Environmental and Experimental Botany, vol. 47, no. 2, pp. 149-155. http://dx.doi. org/10.1016/S0098-8472(01)00122-8.

CANINI, A., ALESIANI, D., D'ARCANGELO, G. and TAGLIATESTA, P., 2007. Gas Chromatography-Mass Spectrometry analysis of phenolic compounds from Carica papaya L. leaf. Journal of Food Composition and Analysis, vol. 20, no. 7, pp. 584-590. http://dx.doi.org/10.1016/j.jfca.2007.03.009. 
CHOW, Y.J. and LIN, C.H., 1991. p-Hydroxy benzoic acid as the major phenolic germination inhibitor of papaya seed. Seed Science and Technology, vol. 19, no. 1, pp. 167-174.

CHRISTOBEL, G.J.R., SHYAM, S.J., PADMANABAN, A.M. and MAHESWARI, A., 2017. Allelopathic potential of Carica papaya leaf extracton growth and biochemical constituents of Phaseolus aureus. International Journal of Recent Advances in Multidisciplinary Research, vol. 4, no. 4, pp. 2555-2560.

DESHMUKHE, P.V., HOOLI, A.A. and HOLIHOSUR, S.N., 2009. Allelopathic potential of Carica papaya on the tobacco cutworm, Spodoptera litura fabricius (lepidoptera:noctuidae). Journal of Advanced Zoology, vol. 30, no. 1, pp. 1-6.

EINHELLIG, F.A., 1996. Mechanism of action of allelochemicals in allelopathy. Agronomy Journal, vol. 88, pp. 886-893. http:// dx.doi.org/10.2134/agronj1996.00021962003600060007x.

FUJII, Y. and AZIZ, M., 2005. Allelopathic effects of some medicinal substances on germination of Amaranthus retrofiexus and Partulaca oleraceae. Belgium: ISHS Acta Horticulterae, pp. 966.

GARIMA and DEVI, M., 2017. Allelopathy in agroforestry: a review. Journal of Pharmacognosy and Phytochemistry, vol. 6, no. 3, pp. 686-688.

GHERARDI, E. and VALIO, I.F.M., 1976. Occurrence of promoting and inhibitory substances in the seed arils of Carica papaya L. Journal of Horticultural Science, vol. 15, no. 1, pp. 1-14. http://dx.doi.org/10.1080/00221589.1976.11514659.

GHORBANLI, M., GRAN, A. and ZOLFAGHARY, A., 2011. The study of Allelopathic potential in three species of Glaucium Mill. on Sinapis arvensis L. Indian Journal of Plant Physiology I Official Publication of the Indian Society for Plant Physiology, vol. 2, no. 1, pp. 321-325.

GOLISZ, A., LATA, B., GAWRONSKI, S.W. and FUJII, Y., 2007. Specific and total activities of the allelochemicals identified in buckwheat. Weed Biology and Management, vol. 7, no. 3, pp. 164-171. http://dx.doi.org/10.1111/j.1445-6664.2007.00252.x.

HAN, C., PAN, K., WU, N., WANG, J. and LI, W., 2008. Allelopathic effect of ginger on seed germination and seedling growth of soybean and chive. Scientia Horticulturae, vol. 116, no. 3, pp. 330-336. http://dx.doi.org/10.1016/j.scienta.2008.01.005.

JAMIL, M., CHEEMA, Z.A., MUSHTAQ, M.N., FAROOQ, M. and CHEEMA, M.A., 2009. Alternative control of wild oat and canary grass in wheat fields by allelopathic plant water extracts. Agronomy for Sustainable Development, vol. 29, no. 3, pp. 475482. http://dx.doi.org/10.1051/agro/2009007.

KATO-NOGUCHI, H., 2011. Barnyard grass-induced rice allelopathy and momilactone B. Journal of Plant Physiology, vol. 168 , no. 10 , pp. 1016-1020. http://dx.doi.org/10.1016/j. jplph.2010.12.021. PMid:21392842.

KHANH, T.D., CHUNG, I.M., TAWATA, S. and XUAN, T.D., 2006. Weed suppression by Passiflora edulis and its potential allelochemicals. Weed Research, vol. 46, no. 4, pp. 296-303. http://dx.doi.org/10.1111/j.1365-3180.2006.00512.x.

KHANH, T.D., CONG, L.C., XUAN, T.D., LEE, S.J., KONG, D.S. and CHUNG, M., 2008. Weed suppressing potential of dodder (Cuscuta hygrophiliae) and its phytotoxic constituents. Weed Science, vol. 56, no. 1, pp. 119-127. http://dx.doi.org/10.1614/ WS-07-102.1.

LERTMONGKOL, S., SAROBOL, E. and PREMASTHIRA, C.U., 2011. Allelopathic effects of mungbean (Vigna radiata) on subsequent crops. Witthayasan Kasetsat Witthayasat, vol. 45, no. 5, pp. 773-779.

MAHARJAN, S., SHRESTHA, B.B. and JHA, P.K., 2007. Allelopathic effects of aqueous extract of leaves of Parthenium hysterophorus $\mathrm{L}$. on seed germination and seedling growth of some cultivated and wild herbaceous species. TheScientificWorldJournal, vol. 5 , pp. 33-39.

MARIANNE, K., MORTEN, S. and BEATE, S., 2000. Ecological effects of allelopathic plants-a review. Silkeborg: National Environmental Research Institute. Nery Technical Report, No. 315.

MARWAT, K.B., KHAN, M.A., NAWAZ, A. and AMIN, A., 2008. Parthenium hysterophorus L., a potential source of bioherbicide. Pakistan Journal of Botany, vol. 40, no. 5, pp. 1933-1942.

MUKHTAR, I., MUSHTAQ, S., HAIDER, M.S. and KHOKHAR, I., 2012. Comparative analysis of autotoxicity in Chenopodium album L., Parthenium hysterophorus L. and Rumex dentatus L. Pakistan Journal of Phytopathology, vol. 24, no. 2, pp. 85-89.

OUDHI, P.A., 2001. Germination and seedling vigour of wheat as affected by allelopathy of some obnoxious weeds. Agricultural Science Digest, vol. 21, no. 4, pp. 275-276.

PELINGANGA, O. and MASHELA, P.W., 2012. Mean dosage stimulation range of allelochemicals from crude extracts of Cucumis africanus fruit for improving growth of tomato plant and suppressing Meloidogyne incognita numbers. J. Agric. Sci., vol. 4, no. 12, pp. 34-45. http://dx.doi.org/10.5539/jas.v4n12p8.

QURESHI, H., ARSHAD, M. and BIBI, Y., 2014. Toxicity assessment and phytochemical analysis of Broussonetia papyrifera and Lantana camara: two notorious invasive plant species. Journal of Biodiversity and Environmental Sciences, vol. 5, no. 2, pp. 508-517

QURESHI, H. and ARSHAD, M., 2017. Dual Potential of allelotoxins for weed control and improved crop growth: A MiniReview. Journal of Environmental and Agricultural Sciences, vol. 12 , pp. $44-53$

REHMAN, H.A., YOUSAF, Z., RASHID, M., YOUNAS, A., ARIF, A., AFZAL, I. and AKRAM, W., 2014. Phytochemical relationship of Euphorbia helioscopia and Euphorbia pulcherrima with Lactuca sativa. Natural Product Research, vol. 30, no. 20, pp. 1-7. PMid:25075544.

REIGOSA, M.J., SANCHEZ-MOREIRAS, A.M. and GONZALEZ, L., 1999. Ecophysiological approach in allelopathy. Critical Reviews in Plant Sciences, vol. 18, no. 5, pp. 577-608. http:// dx.doi.org/10.1080/07352689991309405.

REYES, M.N., PÉREZ, A. and CUEVAS, J., 1980. Detecting endogenous growth regulators on the sarcotesta, endosperm and embryo by paper chromatography on fresh and old seeds of two papaya varieties. The Journal of Agriculture of the University of Puerto Rico, vol. 64, pp. 164-172.

SHANKAR, S.R.M., GIRISH, R., KARTHIK, N., RAJENDRAN, R. and MAHENDRAN, V.S., 2009. Allelopathic effects of phenolics and terpenoids extracted from Gmelina arborea on germination of Black gram (Vigna mungo) and Green gram (Vigna radiata). Allelopathy Journal, vol. 23, pp. 323-332.

SOLTYS, D., KRASUSKA, U., BOGATEK, R. and GNIAZDOWSK, A., 2013. Allelochemicals as bioherbicides present and perspectives. In: A.J. PRICE and J.A. KELTON, eds. Herbicides-current research and case studies in use. London: InTech Publishers, pp. 517-542. http://dx.doi.org/10.5772/56185. 
STEEL, R.G.D. and TORRIE, J.H., 1997. Principles and procedures of statistic. 3rd ed. Singapore: MC Graw Hill Book Co. Inc., pp. 172-177.

TANVEER, A., REHMAN, A., JAVAID, M.M., ABBAS, R.N., SIBTAIN, M., CHAUDHARY, K.M., and AZIZ, A., 2007. Allelopathic potential of Euphorbia helioscopia L. against wheat (Triticum aestivum L.), chickpea (Cicer arietinum L.) and lentil (Lens culinaris Medic.). Turkish Journal of Agriculture and Forestry, vol. 34, no. 1, pp. 75-81. http://dx.doi.org/10.3906/tar-0903-53.

TURK, M.A. and TAWAHA, A.M., 2003. Allelopathic effect of black mustard (Brassica nigra L.) on germination and growth of wild oat (Avena fatua L.). Crop Protection (Guildford, Surrey), vol. 22, no. 4, pp. 673-677. http://dx.doi.org/10.1016/S02612194(02)00241-7.

WOLF, R.B., SPENCER, G.F. and KWOLEK, W.F., 1984. Inhibition of velvetleaf (Abutilon theophrasti) germination and growth by Benzyl isothiocyanate, a natural toxicant. Weed Science, vol. 32, no. 5, pp. 612-615. http://dx.doi.org/10.1017/ S004317450005966X.

YAMADA, K., HIROSE, K., SHIGEMORI, H. and HASEGAWA, K., 2010. Plant growth promotive allelochemicals. Japan: The Society of Synthetic Organic Chemistry, vol. 68, pp. 551-562. 